\title{
PROTOTIPO PICK AND PLACE MACHINE
}

PICK AND PLACE MACHINE PROTOTYPE

José Antonio Velásquez Costa ${ }^{1}$

RECEPCIÓN: 15 DE NOVIEMBRE DEL 2019

ACEPTACIÓN: 05 DE DICIEMBRE DEL 2019

\section{RESUMEN}

Pick and Place Machine es un proyecto de automatización industrial que busca reducir los costos y los tiempos de producción en el proceso de recoger y abastecer de insumos, materiales, piezas y, en general, cualquier producto. De esa manera, contribuye con el incremento de la eficiencia en diversas industrias. Una pinza plana neumática recogerá el material por accionamiento de los dos cilindros neumáticos, que se extenderán uno después de otro. Luego, transportará el producto hacia la derecha en forma lineal en un recorrido de $100 \mathrm{~mm}$, donde una pinza se abrirá y soltará el producto para luego regresar a su estado inicial de reposo. Todo el proceso está controlado mediante un PLC (controlador lógico programable). El proyecto fue desarrollado en el curso Procesos de Manufactura Asistida por Computadora II de Ingeniería Industrial de la Universidad Ricardo Palma, por los estudiantes de Valeria Avalo Lazo, Diana Huamán Zenteno, Gabriel Osorio Castro, Oscar Pacheco Baca y Milagros Vera Román. Este proyecto fue ganador del primer puesto en el concurso de la XXV Feria de Creatividad e Innovación Tecnológica Industrial - FECITIN, realizado en noviembre del 2019.

Palabras clave: PLC, cilindro neumático, prototipo, automatización.

\begin{abstract}
Pick and Place Machine is an industrial automation project that seeks to reduce costs and also production times in the process of collecting and supplying inputs, materials, parts and in general any product, contributing to the increase of efficiency in various industries. A pneumatic flat clamp will pick up the material by actuating the 2 pneumatic cylinders that will extend one after the other, then transport the product to the right in a linear way in a $100 \mathrm{~mm}$ path where a clamp will open and release the product and then return to Your initial state of rest. The whole process is controlled by a PLC (programmable logic controller). The project was developed in the Computer Aided Manufacturing Processes Course II of Industrial Engineering at Ricardo Palma University, by the students of Valeria Avalo Lazo, Diana Huamán Zenteno, Gabriel Osorio Castro, Oscar Pacheco Baca and Milagros Vera Román. This project was the winner of the first place in the Contest of the XXV Fair of Creativity and Industrial Technological Innovation - FECITIN done in November 2019.
\end{abstract}

Keywords: PLC, pneumatic cylinder, prototype, automation. 


\section{INTRODUCCIÓN}

Hoy en día, el término automatización industrial se refiere al uso de tecnologías para controlar procesos que, hasta hace algunos ańos, se hacían de manera manual. Esto limita la intervención humana, lo que permite elevar la calidad del producto final; incrementar la producción, dado que los procesos serán más rápidos, y eliminar riesgos para las personas.

Las tecnologías que han permitido el desarrollo de la automatización son la neumática, hidráulica, electrónica, robótica y los PLC (programmable logic controller).

Una de las razones por las que las empresas deben automatizarse es para continuar estando presentes en el mercado, que tiene cada vez más clientes exigentes y que están en continua búsqueda de productos o servicios de alta calidad, con un menor costo y en tiempos más reducidos.

Pick and place machine es un proyecto que permite el rápido trasporte de materiales pesados a una distancia específica, con lo que reduce tiempos y el esfuerzo de trabajo excesivo al que están sometidos los trabajadores, además de asegurar la conservación de los elementos que moviliza, mantener un ritmo constante de operación y desplazarse sin interrupciones. Solo se le debe dar el correcto mantenimiento de manera constante para evitar paros durante la producción cuando sea aplicado en las empresas.

Para realizarlo se hace uso de seis sensores magnéticos. Los que son de tipo discreto están conectados a las entradas del PLC (controlador lógico programable), mientras que las electroválvulas se encuentran conectadas en las salidas del PLC. Pick and Place machine ayuda a escoger con rapidez el material o producto, ya que reduce el empleo de recurso humano para su realización.

\section{ANTECEDENTES}

El Perú es un país de múltiples industrias, que han ido aumentando desde hace 2 décadas, por lo que hay, cada vez más, empresas que producen y dan servicios. Por otro lado, las que tienen un mayor rango de sostenibilidad son las que cuentan con más de doce trabajadores, que producen más del 85\% del total de la manufactura y estas se encuentran en la ciudad de Lima. Por el contrario, en las ciudades de las provincias, la mayoría son industrias pequeñas con tecnología baja.

En general, actualmente, la industria manufacturera está en auge, a pesar de que aún no se emplea tecnología de punta, como sucede en países europeos. Sin embargo, existen iniciativas por parte de empresarios de países desarrollados que quieren invertir y traer su tecnología para aprovecharla en nuestra industria manufacturera.

\section{OBJETIVO}

El proyecto tiene, como objetivo principal, reducir los tiempos de producción y costos asociados en el proceso de recoger y abastecer de insumos, materiales, piezas y, en general, cualquier producto, para contribuir con el incremento de la eficiencia en una línea de producción.

\section{METODOLOGÍA}

La automatización industrial es la utilización de un conjunto de técnicas que se suman al uso de elementos computarizados, que tienen como finalidad controlar equipos o máquinas que forman parte de los procesos de manufactura o industriales, con la intención de sustituir a operarios. 
Su alcance supera la simple mecanización de los procesos de manufactura, ya que disminuye el esfuerzo físico que realiza cada operario. Además, la automatización también reduce ampliamente la necesidad sensorial y mental de las personas que están ligadas al proceso de producción, debido a que existen sensores que pueden realizarlas con mucha mayor eficiencia. Para [1], «la automatización como una disciplina de la ingeniería tiene como herramienta, el uso de sensores y actuadores, controladores lógicos programables (PLC's), sistemas de control y supervisión (SCADA) y aplicaciones para supervisar y controlar en tiempo real las operaciones de plantas o procesos industriales».

El prototipo Pick and Place Machine está formado por dos niveles esenciales:

- Nivel de mando: está dado por el cerebro del proceso automatizado, es decir, por el PLC, que está programado con el software TIA PORTALv14. La programación en lenguaje ladder o escalera permite establecer las secuencias, tiempos y condiciones que deben cumplirse en el proceso productivo. De esta manera, se facilita el control de calidad, la reducción de trabajo y el incremento de la productividad.

- Nivel operativo: son los dispositivos que provocan que un equipo o una máquina se mueva y realice la operación deseada en función a la programación realizada en el PLC. Está constituido por dos cilindros planos neumáticos de doble efecto; una pinza neumática; tres electroválvulas de 5/2 monoestable de 3/8" 24VDC; seis sensores magnéticos para cilindros; un PLC de marca SIEMENS, modelo S71200 CPU 1214C; una caja de pulsadores (start, stop y reset); seis racores rectos para mangueras de $6 \mathrm{~mm}$; un cable de ethernet y conectores rápidos de salida de $6 \mathrm{~mm}$ de diámetro.

\section{MARCO TEÓRICO}

\section{Válvulas electroneumáticas}

Las válvulas electroneumáticas tienen como fin principal orientar y distribuir el aire comprimido que sale del compresor para que sea empleado en un circuito electroneumático. Son capaces de regular el paso del aire o impedir que ello ocurra. Estos dispositivos preparan el camino que debe recorrer el aire comprimido a una presión de 5 bar. Para clasificar las válvulas, se pueden mencionar cuatro distintos tipos según su función: reguladoras, direccionales, secuenciales y de bloqueo. Cada una tiene un trabajo particular que las diferencia de las demás. En el proyecto, se emplean electroválvulas direccionales, dado que su objetivo principal es distribuir aire comprimido.

Todas las electroválvulas mencionadas anteriormente cuentan con distinta cantidad de vías y de posiciones, y la suma de las roscas internas que posee corresponde a la cantidad de vías que tiene el dispositivo. Las electroválvulas seleccionadas para el proyecto fueron 5/2 monoestables (cinco vías y dos posiciones) de $24 \mathrm{VDC}$, con accionamiento por resorte. Para [2],

este tipo de válvulas tienen 2 conexiones de escape, esto es dado que su construcción interna proporciona un escape a cada salida en lugar de un escape común. La aplicación más usual de la electroválvula $5 / 2$ monoestable es controlar el avance y retroceso de un cilindro neumático de doble efecto, en una posición de la válvula el cilindro se extiende y en la otra regresa a su posición inicial.

\section{Sensores magnéticos}

Son dispositivos electrónicos que se encargan de detectar campos magnéticos de los imanes o corrientes eléctricas. Internamente, poseen un par de láminas de material ferromagnético, alojadas en 
una capsula que es atraída por un campo magnético, con lo que se cierra un circuito eléctrico [3]. Se caracterizan por su robustez y eficiencia. Su función en el proyecto es detectar la posición de cada cilindro neumático.

\section{Controlador lógico programable (PLC)}

Es un dispositivo de control que monitorea la condición de los sensores que están conectados en sus inputs y, según el programa ladder almacenado en su memoria, controla el estado de los dispositivos que están conectados en sus outputs. El PLC empleado es un Siemens, modelo S71200.

\section{Actuadores lineales (Cilindros neumáticos)}

Los actuadores lineales son dispositivos que convierten la energía del aire comprimido en trabajo mecánico por medio de un movimiento lineal de vaivén o de motores. Los actuadores neumáticos se clasifican en dos grupos: cilindros y motores.

Para [4],

los cilindros neumáticos transforman la energía neumática en trabajo mecánico de movimiento rectilíneo, que consta de carrera de avance y carrera de retroceso. Existen diferentes tipos de cilindros neumáticos. Según el modo en que se realiza el retroceso del vástago, los cilindros se dividen en tres grupos cilindros de simple efecto, cilindros de doble efecto, cilindro de rotación.

Los cilindros neumáticos empleados en el proyecto son de doble efecto y cilindro de rotación.

- Cilindros de doble efecto

Los cilindros de doble efecto se emplean especialmente en los casos en que el émbolo tiene que realizar una misión también al retornar a su posición inicial, ya que hay un esfuerzo neumático en ambos sentidos. Se dispone de una fuerza útil en ambas direcciones [5].

\section{DISEÑO DEL PROYECTO}

«El software de diseño CAD se usa en casi todas las industrias. Los sistemas de diseño CAD 2D y 3D se utilizan para dibujar de forma visual diseños de plantas o de fábricas» [6]. Para el diseño del proyecto, se emplearon herramientas de desarrollo de prototipos digitales, como es el software SolidWorks. La figura 1 muestra el diagrama de despiece del Pick and Place Machine. Asimismo, la figura 2 muestra las distintas vistas del prototipo.

Figura 1. Diagrama de despiece del Pick and Place Machine. Elaboración propia.

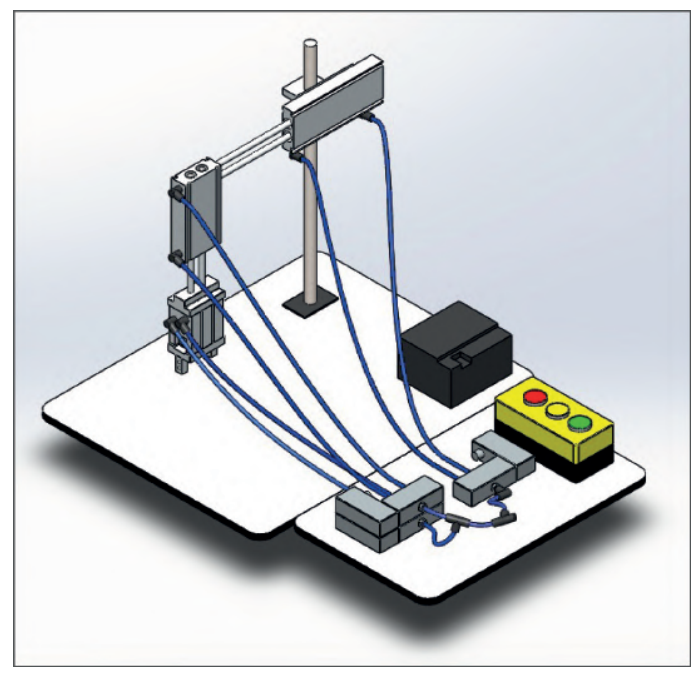




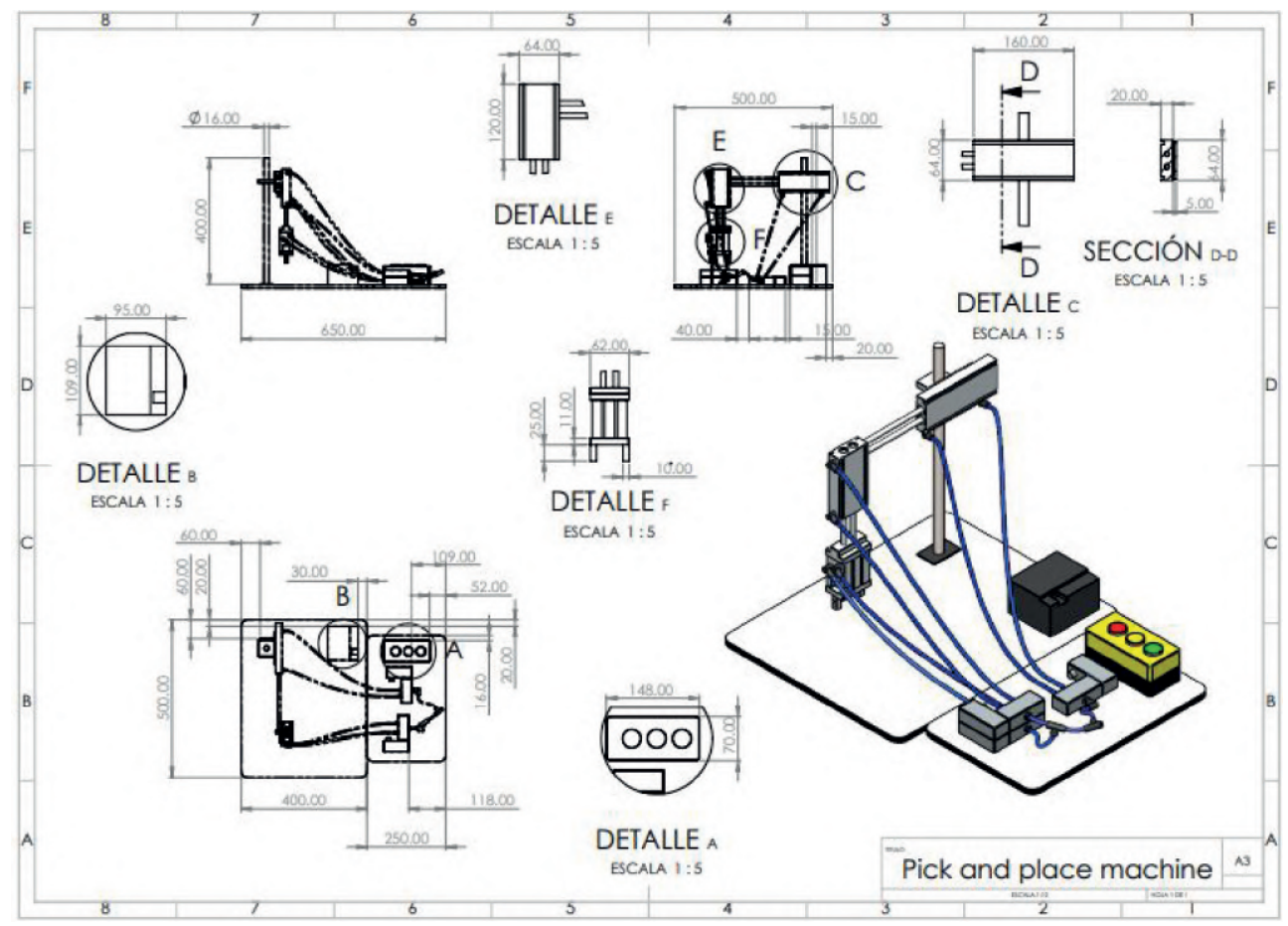

Figura 2. Vistas del Pick and Place Machine. Elaboración propia.

\section{PROGRAMACIÓN DEL PROYECTO}

La programación del proyecto se realizó en el PLC, con el software TIA Portal v14. Se empleó el lenguaje ladder para que el proceso se realice de manera automática con sólo presionar un pulsador. A continuación, se detalla la programación.

\section{Segmento 1:}

Comentario

"Start"


INDUSTRIAL

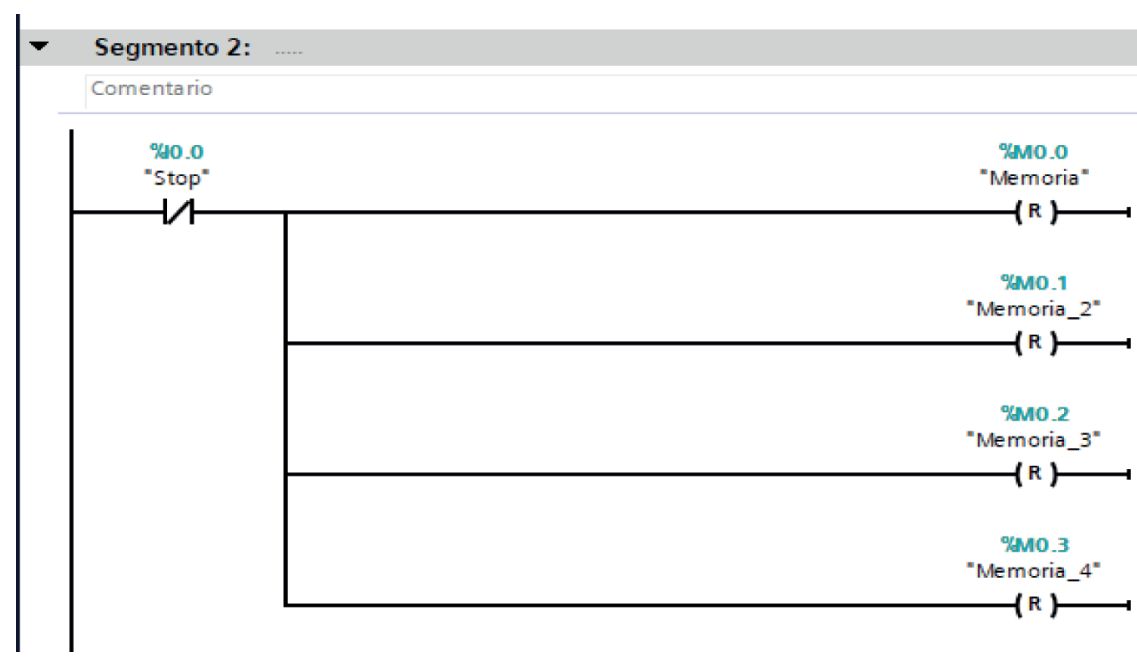

Segmento 3:

Comentario

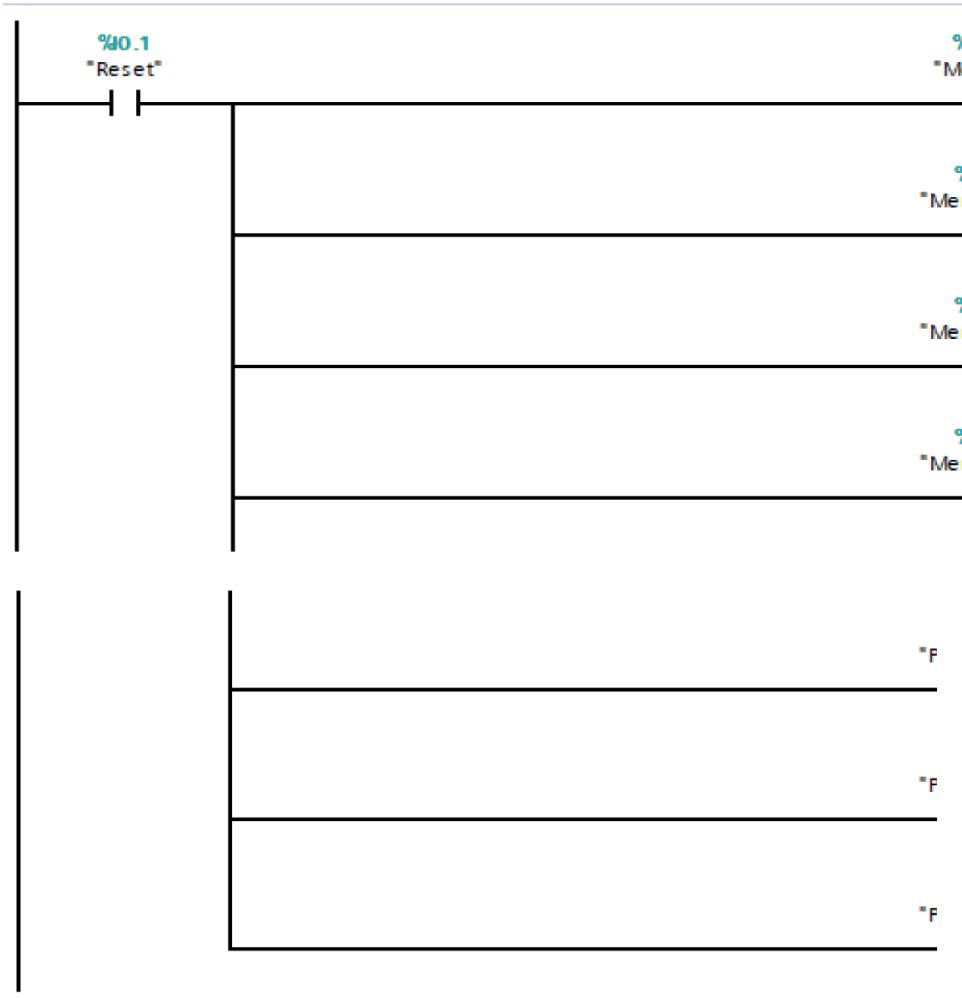

\%(No 0

"Memoria"

( R )

\%MO 1

Memoria_2"

$(R)$

\%MO 2

Memoria_3"

$(R)$

\%MNO_3

Memoria_4"

$(R)$ 


\section{Segmento 4:}

Comentario

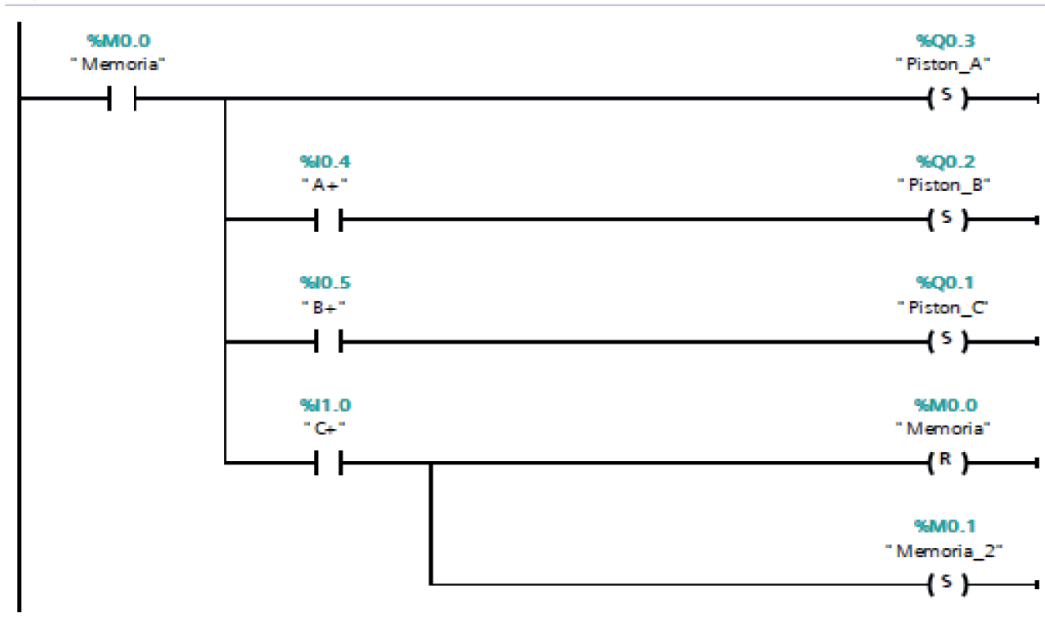

\section{Segmento 5:}

Comentario

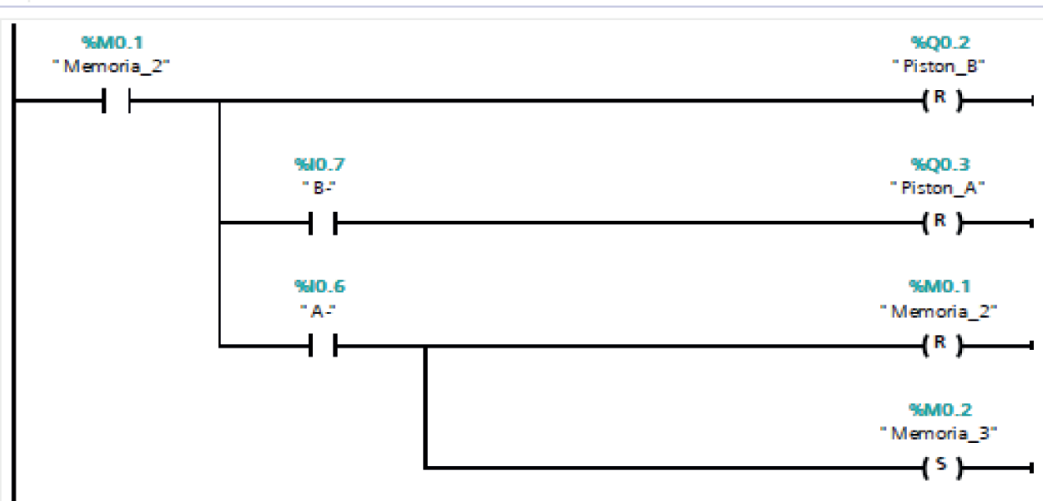

\section{Segmento 6:}

Comentario

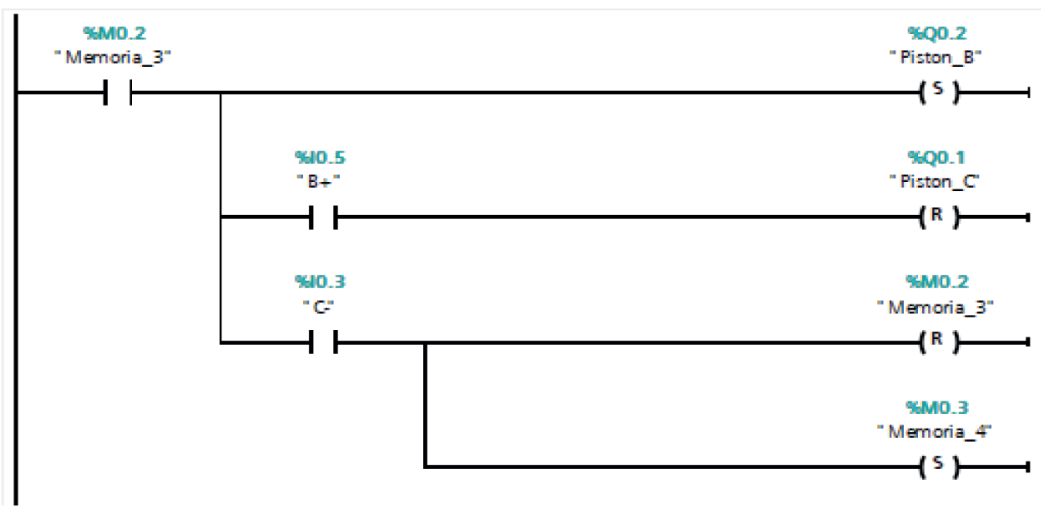




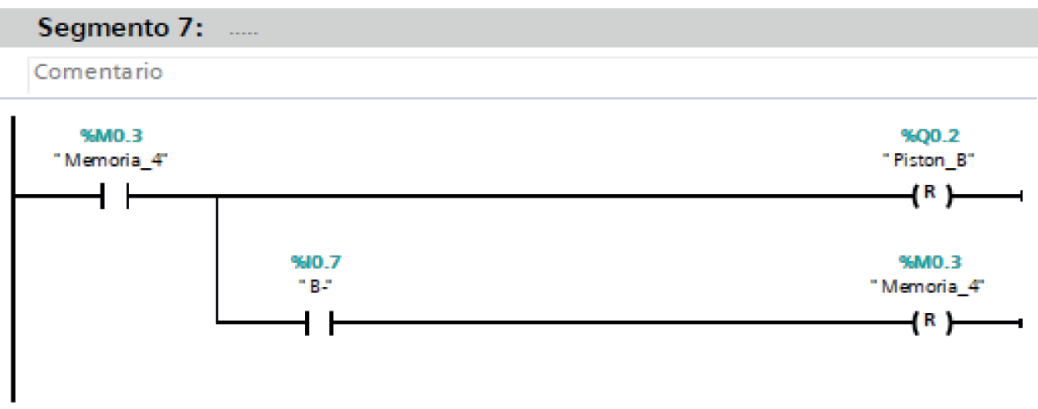

\section{FUNCIONAMIENTO}

Una vez encendido el prototipo, a través del pulsador verde (start), comienza el proceso, en el cual uno de los cilindros lineales se extenderá para coger el producto que debe ser trasladado. Cuando el sensor magnético detecte su posición final, se cerrará la pinza neumática. Luego, el mismo cilindro neumático se retrae y procede a extenderse el segundo cilindro neumático lineal, para que luego se extienda el primero y la pinza se abra. De esa manera, se deja el producto en la posición de destino. Finalmente, los cilindros lineales retornan a su posición inicial. Existe, además, un pulsador rojo (stop) para detener el proceso y un pulsador amarillo (reset) para que los cilindros y pinzas retornen a su posición inicial de reposo. La figura 3 muestra el prototipo implementado y la figura 4, la premiación por haber obtenido el primer puesto en el FECITIN 2019-II

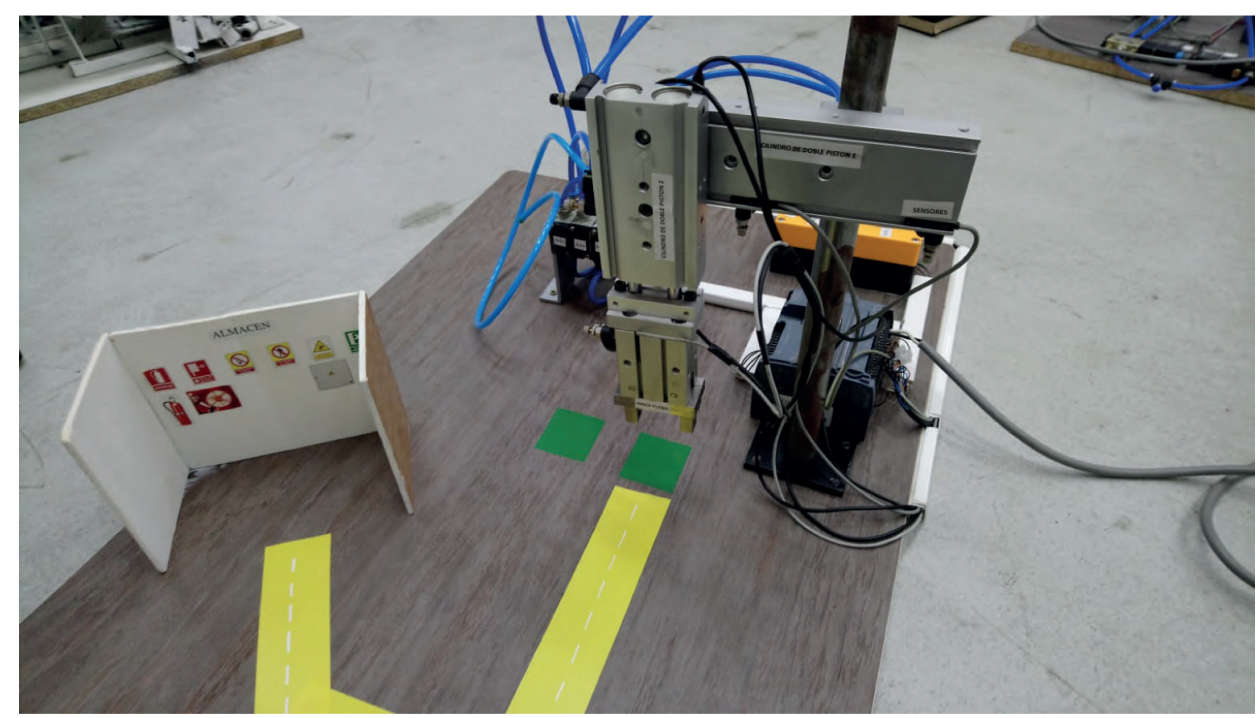

Figura 3. Prototipo Pick and Place Machine. Elaboración propia. 


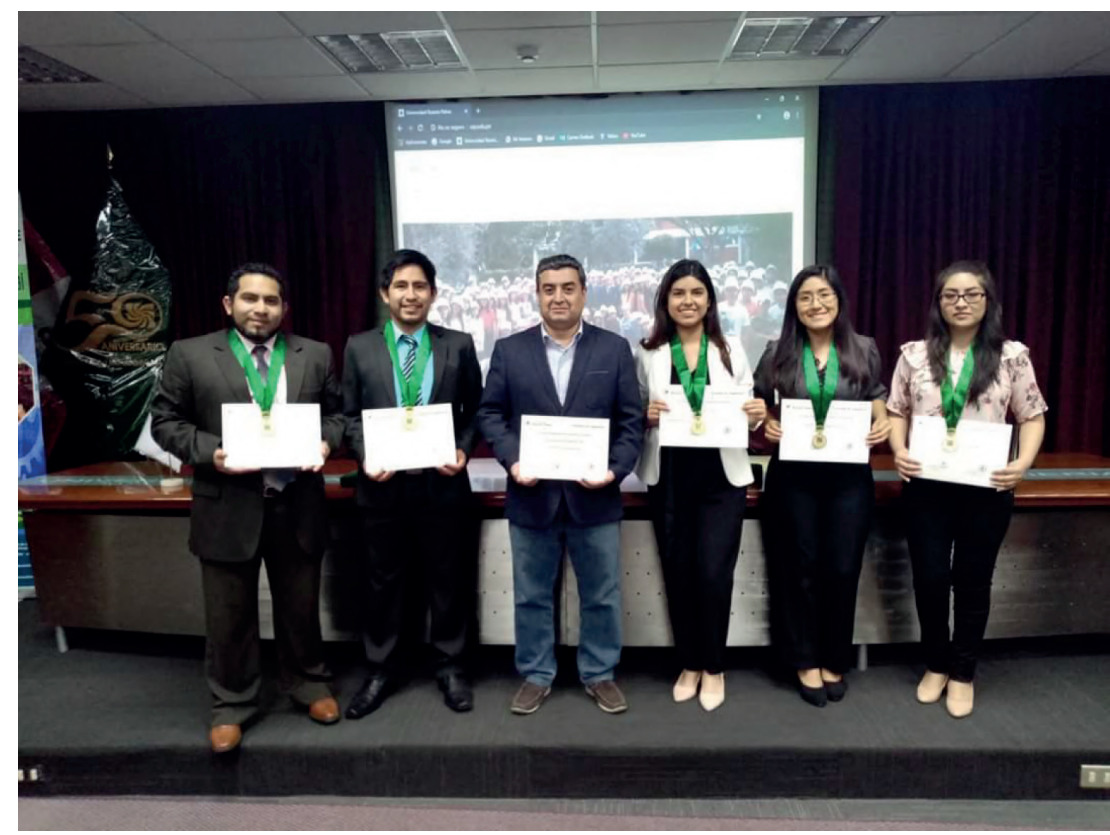

Figura 4. Premiación por haber obtenido el primer puesto en el FECITIN 2019-II. Elaboración propia.

\section{CONCLUSIONES}

- Dado que la automatización estandariza el proceso, se puede garantizar la disminución de tiempos en el proceso de transporte de piezas u objetos, y el aumento de la productividad.

- La reducción de los tiempos de traslado de materiales también se verá reflejada en los costos asociados a este proceso.

- El prototipo también optimiza el proceso en términos de mano de obra, tiempos de selección y eliminación de reproceso. Además, contribuye con el incremento de la eficiencia en una línea de producción.

\section{BIBLIOGRAFÍA}

[1] W. Bolton, Programmable Logic Controller, United States of America: Elsevier Science, 2015.

[2] S. Gallardo, Configuración de instalaciones domóticas y automáticas, Madrid: Paraninfo, 2019.

[3] J. Velásquez, «Los sensores en la producción,» Revista de la Facultad de Ingeniería de la Universidad Ricardo Palma, 2005.

[4] J. Entrena, Instalación de equipos y elementos de sistemas de automatización industrial, Málaga: IEC Editorial, 2015.

[5] A. Serrano, Neumática Práctica, Madrid: Paraninfo, 2009.

[6] J. Velásquez, "Implementación de un prototipo de una dispensadora y envasadora de chocolates,» Revista Industrial Data de la Universidad Nacional Mayor de San Marcos, 2009. 
